\title{
ARTÍCULOS ORIGINALES \\ Conocimientos y prácticas en prevención de caries y gingivitis en educadores de párvulos, JUNJI, Santiago
}

\author{
PAMELA VÁSQUEZ ${ }^{(1)}$
}

\section{RESUMEN}

Objetivos: La caries dental y la gingivitis son prevalentes en la población infantil. El propósito de este estudio fue describir los conocimientos que poseen los educadores de párvulos de la provincia de Santiago, en la prevención de caries y gingivitis de los preescolares, así como las prácticas declaradas que realizan durante la jornada escolar, que pudiesen favorecer el control de éstas.

Método: Se realizó un estudio descriptivo, transversal, y cuantitativo, con un muestreo probabilístico estratificado proporcional, por conglomerados, bietápico para seleccionar una muestra de 209 profesionales, de la Junta Nacional de Jardines Infantiles de Santiago, quienes respondieron un cuestionario auto-administrado, previamente validado. (Confiabilidad 0.78, alpha de Cronbach).

Resultados: El nivel de conocimientos fue catalogado como bueno (0.69, intervalo de confianza 0.67-0.70). En cuanto a las prácticas preventivas, en el 98\% de los establecimientos se realiza cepillado dental. En relación al tipo de alimentos consumidos, priman las frutas y leche, con un $52.3 \%$ y un $58.3 \%$ respectivamente.

Los tópicos con menor porcentaje de respuestas correctas correspondieron a uso de fluoruros tópicos, edad de inicio y frecuencia de cepillado.

Conclusión: Existe un nivel adecuado de conocimientos en los docentes, lo que constituye una herramienta valiosa para generar y mantener cambios conductuales favorables a la salud bucal. En el ámbito de las prácticas declaradas, prácticamente la totalidad de los educadores consultados reporta que sus pupilos realizan el cepillado dentario, con una frecuencia de dos veces al dia, siendo concordante con lo recomendado como efectivo en la literatura especializada.

Palabras clave: caries dental, gingivitis, prevención, preescolar.

\section{ABSTRACT}

\section{KNOWLEDGE AND PREVENTIVE PRACTICES REGARDING CAVITIES AND GINGIVITIS AMONG KINDERGARTEN TEACHERS FROM THE NATIONAL BOARD OF KINDERGARTENS OF SANTIAGO.}

Purposes: Dental cavities and gingivitis are prevalent among the pediatric population. The purpose of the present work was to describe the knowledge in cavity and gingivitis prevention among kindergarten teachers in the province of Santiago and to assess their declared practices during the full school day which might prevent such conditions.

\footnotetext{
(1) Departamento de Salud Bucal. Ministerio de Salud. Mac-Iver 541. Santiago. Chile. pvasquez@minsal.cl
} 
Methods: A cross-sectional, descriptive, and quantitative study was conducted with proportional stratified probabilistic cluster two-stage to select a sample of 209 professionals from the National Board of Kindergartens of the province of Santiago, who answered a previously validated selfadministered survey (Cronbach's alpha reliability of 0.78). .

Results: The degree of knowledge was rated as good (0.69, Confidence Interval 0.67-0.70). As for preventive practices, toothbrushing was carried out in $98 \%$ of the facilities. With regard to the kind of food consumed, fruits and milk prevailed, with $52.3 \%$ and $58.3 \%$ respectively.

The topics with the lowest correct answer rates were those regarding the use of topical fluoride, the onset of toothbrushing habits and toothbrushing frequency.

Conclusions: There is an adequate degree of knowledge among teachers. Such knowledge constitutes a valuable tool to generate and sustain behavioral changes favoring oral health. In the field of declared practices, practically all the teachers surveyed reported that their pupils toothbrush on a twice a week basis, thus being in accordance with the recommended effective toothbrushing practice in the specialized literature.

Keywords: dental cavities, gingivitis, prevention, preschool.

\section{INTRODUCCIÓN}

La alta prevalencia de enfermedades bucodentales en la población ${ }^{1}$, referida a caries dentales y enfermedades gingivales y periodontales, constituye un problema de salud pública en Chile. Aún cuando no existan estudios nacionales publicados, específicos para preescolares, se extrapola la realidad en dentición definitiva, o mixta a lo que ocurre en dentición temporal. Es así como, según el estudio reciente (2007) realizado por el Ministerio de Salud, se tiene como referencia que el $29.64 \%$ de los niños de 6 años estudiados en la muestra, estaba libres de caries ${ }^{2}$, meta distante del $50 \%$ propiciado por la Organización Panamericana de la Salud para los niños de 5 a 6 años de edad. En el 70.36\% restante, con daño por caries, el índice ceod, que mide la historia de daño por caries en piezas temporales, fue de 3.71 .

En Escocia, el promedio de dientes temporales cariados, extraídos u obturados para un niño de 5 años es de 2.7, en comparación con el 1.6 de un niño de la misma edad del Reino Unido ${ }^{3}$ ). La Encuesta Nacional Española de Salud Bucal en preescolares $2007^{4}$ reportó una prevalencia de caries de $17 \%$ en los niños de 3 años y de $26 \%$ en los de 4 años.

Esta situación, sumada a los recursos humanos y físicos insuficientes para su abordaje, hace necesario anticiparse al daño mediante medidas de prevención en edades tempranas de la vida, de modo de impedir su aparición y/o aminorar los niveles de severidad.

En Chile, las medidas preventivas de salud se realizan fundamentalmente a través de los consultorios de atención primaria de salud, dependientes de los Departamentos de Salud de los Municipios o de las Corporaciones Municipales.

Sin embargo, la demanda asistencial curativa ha dejado la prevención primaria en salud bucal relegada a un segundo plano. Por esto es necesario buscar estrategias que permitan llegar con los mensajes educativos a la mayoría de la población, de modo que las personas puedan tomar las mejores decisiones y adquirir hábitos favorables para su salud bucal.

La Junta Nacional de Jardines Infantiles (JUNJI) es un organismo autónomo que brinda atención a los menores de los sectores más desposeídos del país. Atiende, en la Región Metropolitana, a 30.000 niños aproximadamente, otorgándoles educación parvularia integral ${ }^{5}$. Debido a la prolongada fase de tiempo que las educadoras de párvulos acompañan a los menores en su desarrollo, y debido también a la influencia que ejercen sobre ellos y sus padres, es que estas docentes tienen la oportunidad de cultivar hábitos, conocimientos y prácticas favorables a la salud bucal. 


\section{MATERIAL Y MÉTODO}

La investigación correspondió a un estudio descriptivo, transversal y cuantitativo, realizado en los Jardines Infantiles de la Junta Nacional de Jardines Infantiles de Santiago. El marco muestral lo constituyó el registro de 136 jardines infantiles de la entidad mencionada, con nombre, dirección, teléfono y código disponible en la base de datos del Departamento de Salud Bucal del Ministerio de Salud, al 2007. El criterio de inclusión para los educadores de párvulos fue: ser parvularia(o) titulada(o), haber estudiado en un establecimiento de educación superior nacional, trabajar en un jardín infantil de la entidad, en la provincia de Santiago en el nivel medio o transición y ser de nacionalidad chilena. Esto último para no contaminar los resultados con información obtenida por otros sistemas educacionales y/o culturales.

Se usó un método de muestreo probabilístico estratificado proporcional, por conglomerados, bietápico. La estratificación se realizó por comuna en base al Índice de Desarrollo Humano (IDH) comunal. Esta metodología propuesta por la ONU, califica la calidad de vida de la población a través de tres indicadores: esperanza de vida al nacer, nivel educacional y nivel de vida ${ }^{6}$.El tamaño de la muestra se determinó en base al valor más alto de $\mathrm{p}(0.5)$ por no existir estudios previos al respecto que permitan obtener la estimación del parámetro, con un error estándar de 0.015 . El resultado determinó un tamaño de muestra de 121 jardines infantiles para acceder a las y los 209 parvularios que conforman la unidad de análisis. La selección de la muestra se realizó mediante números aleatorios del programa Excel.

El instrumento de recolección de datos (cuestionario), fue confeccionado especialmente, cimentado en la información relevante pesquisada en la literatura especializada ${ }^{3,7-9}$ en base a 29 preguntas cerradas, precodificadas, que abarcaron los ámbitos de conocimientos y prácticas (Figuras 1 y 2), y cinco preguntas abiertas para datos biodemográficos. Previo a su aplicación, fue validado por tres expertos profesionales: una asistente social, encargada de promoción del Servicio Metropolitano Occidente; una educadora de párvulos, egresada de la Universidad de Chile, con 20 años de experiencia en el trabajo con párvulos, en diversas instituciones, públicas y privadas, y una odontóloga encargada de promoción de la salud del Servicio de Salud del Libertador Bernardo O’Higgins. El instrumento se piloteó en nueve educadoras de párvulos, para ajustar lenguaje y coherencia, lo que implicó eliminar dos preguntas, y reformular tres. La confiabilidad fue de 0.78 medida con alpha de Cronbach.

Para analizar los resultados se elaboró una escala de intervalos para el área temática de conocimientos (Figura 3), basándose en estudios similares $^{10,11}$.

Figura 1. Dimensión de conocimientos desglosada por temas y número de preguntas asociadas.

\begin{tabular}{|l|l|l|}
\hline $\begin{array}{l}\text { Dimensión } \\
\text { conocimientos }\end{array}$ & Subdimensión & $\mathbf{N}^{\mathbf{0}}$ preguntas \\
\hline Caries & Definición & 1 \\
\hline & Mecanismo de producción & 2 \\
\hline & Prevención por autocuidado & 1 \\
\hline Dieta cariogénica & Atributos de la dieta que la hacen más dañina & 2 \\
\hline Uso de fluoruros & Existencia o no de beneficios & 1 \\
\hline & Vías de administración & 1 \\
\hline & Uso en párvulos a través de pastas dentales & 5 \\
\hline Gingivitis & Definición & 1 \\
\hline & Mecanismo de producción & 1 \\
\hline Higiene bucal & Edad de inicio & 1 \\
\hline & Frecuencia de cepillado & 1 \\
\hline & Características del cepillo & 3 \\
\hline
\end{tabular}


Figura 2. Dimensión de prácticas desglosada por temas y número de preguntas asociadas.

\begin{tabular}{|l|l|l|}
\hline $\begin{array}{l}\text { Dimensión } \\
\text { Prácticas }\end{array}$ & Subdimensión & $\mathbf{N}^{\mathbf{0}}$ preguntas \\
\hline Dieta & Colaciones sólidas & 1 \\
\hline & Colaciones líquidas & 1 \\
\hline & Reforzador primario & 1 \\
\hline Higiene bucal & Cepillado en el establecimiento & 1 \\
\hline & Frecuencia & 1 \\
\hline & Elección cepillo de dientes & 1 \\
\hline & Uso y tipo de pasta dental & 2 \\
\hline & Técnica de cepillado enseñada & 1 \\
\hline
\end{tabular}

Figura 3. Escala de evaluación del nivel de conocimientos.

\begin{tabular}{|l|l|}
\hline Nivel & Rangos \\
\hline Insuficiente & $0.00-0.50$ \\
\hline Suficiente & $0.51-0.67$ \\
\hline Bueno & $0.68-0.84$ \\
\hline Muy bueno & $0.85-1.0$ \\
\hline
\end{tabular}

Para el área de prácticas en salud bucal, las acciones de prevención en base a instrucción y realización de cepillado, uso de fluoruros tópicos en la forma de pastas dentales infantiles, y colaciones no cariogénicas, sólo se describieron en base al porcentaje de realización.

El análisis de los datos obtenidos fue cuantitativo, de estadística descriptiva, recolectando la información, en primera instancia en planilla Excel, y posteriormente transfiriéndolos al programa STATA 9.0, para cada variable definida previamente.

\section{RESULTADOS}

Las respuestas fueron anónimas y voluntarias, por lo que no se obtuvo ninguna información de las personas que no devolvieron el cuestionario.

De la muestra conformada por 209 educadoras de párvulos de los jardines clásicos de la provincia de Santiago, provenientes de 121 jardines infantiles, se obtuvo respuesta de 180 parvularias $(86.1 \%)$, pertenecientes a 105 establecimientos, cantidad considerada adecuada a las necesidades del estudio, en base a información de la literatura que reporta tasas de respuestas a este tipo de cuestionarios, aplicados en poblaciones controladas, de un $85 \%$, siendo aceptables valores de $75 \%$ y más ${ }^{12,13}$.

Como muestra la Tabla 1 poco más de la mitad de los educadores (51.7\%) tiene entre $49 \mathrm{y}$ 62 años de edad, siendo el rango etáreo de las personas consultadas de 24 a 62 años. En cuanto al sexo de los encuestados, hubo solo 1 varón en el grupo que respondió el cuestionario.

Dimensión Caries. Un $77.8 \%$ de las parvularias conocía la definición conceptual de caries dental. El 95\% mostró conocer el mecanismo de producción de la caries dental, lo que es consistente con el conocimiento reportado de placa bacteriana (91.7\%). Sin embargo, solo un 53.3\% identificó las formas de prevenir la enfermedad. Este punto, por tanto, debe ser reforzado, para la prevención de caries dental a nivel preescolar.

Dimensión Dieta Cariogénica. Al consultar por uno de los factores que intervienen en la producción de caries dental, esto es, el tipo de alimentación consumida, su frecuencia de ingesta, los resultados fueron buenos en términos generales: un 75.6\% identificó los atributos de la 
Tabla 1. Características de educadores de párvulos según IDH, edad y experiencia profesional, en años. Santiago 2007.

\begin{tabular}{|l|l|l|l|l|l|l|l|l|}
\hline & \multicolumn{3}{l}{ IDH Muy Alto } & \multicolumn{2}{l|}{ IDH Alto } & \multicolumn{2}{l|}{ IDH Medio } & \multicolumn{2}{l|}{ Total } \\
\hline & $\mathrm{N}$ & $\%$ & $\mathrm{~N}$ & $\%$ & $\mathrm{~N}$ & $\%$ & $\mathrm{~N}$ & $\%$ \\
\hline Edad & & & & & & & & \\
\hline $21-34$ & 13 & 17.3 & 16 & 18.6 & 2 & 11.1 & 31 & 17.2 \\
\hline $35-48$ & 20 & 26.7 & 28 & 31.4 & 8 & 44.4 & 56 & 31.1 \\
\hline $49-62$ & 42 & 56 & 43 & 50 & 8 & 44.4 & 93 & 51.7 \\
\hline $\begin{array}{l}\text { Experiencia } \\
\text { profesional }\end{array}$ & & & & & & & & \\
\hline No especifica & - & - & 2 & 2.3 & - & - & 2 & 1.1 \\
\hline $0-9$ & 15 & 20 & 28 & 32.2 & 3 & 16.7 & 46 & 25.6 \\
\hline $10-19$ & 13 & 17.3 & 15 & 17.2 & 6 & 33.3 & 34 & 18.9 \\
\hline $20-29$ & 24 & 32 & 28 & 32.2 & 7 & 38.9 & 59 & 32.8 \\
\hline $30-39$ & 23 & 30.7 & 14 & 16.1 & 2 & 11.1 & 39 & 21.6 \\
\hline Total & $\mathbf{7 5}$ & $\mathbf{1 0 0} \%$ & $\mathbf{8 7}$ & $\mathbf{1 0 0} \%$ & $\mathbf{1 8}$ & $\mathbf{1 0 0 \%}$ & $\mathbf{1 8 0}$ & $\mathbf{1 0 0 \%}$ \\
\hline
\end{tabular}

dieta consumida que favorecen el desarrollo de caries, y un $85 \%$ demostró reconocer la capacidad dañina de las bebidas líquidas con contenido azucarado, y/o contenido acídico.

Dimensión Uso de Fluoruros. Para evaluar las medidas de prevención de caries dental, del tipo individual, se consultó por el fluoruro y su aplicación en la forma de pastas dentales.

El 97.2\% de los consultados reconoció la existencia de beneficios para los dientes, al usar fluoruro. Sin embargo, sólo un $29.4 \%$ fue capaz de identificar las formas de obtener fluoruro, ya sea por vía sistémica o tópica.

Un 37.8\% dijo conocer la diferencia entre las pastas dentales infantiles y de adulto. E1 $51.7 \%$ de los encuestados acertó en la edad correcta de inicio del uso de pasta dental fluorurada. Mientras que el $82.2 \%$ tenía clara la dosificación apropiada de dentífrico fluorurado para los párvulos de tres años. Un porcentaje superior $(86.1 \%)$ declaró conocer la necesidad de supervisar el cepillado de dientes para vigilar técnica, cantidad de pasta usada y su eliminación al finalizar.

Dimensión Gingivitis. Un 92.2\% de las personas que contestaron el cuestionario, reconoció la definición conceptual de gingivitis. El mecanismo de producción fue respondido en forma acertada por un $88.3 \%$ de las educadoras.
Dimensión Higiene Bucal. Al consultar por la edad de inicio del cepillado de dientes, se obtuvo un $74.4 \%$ de aciertos. En cuanto al conocimiento existente respecto de la frecuencia adecuada de cepillado diario en preescolares, sólo un 13.3\% tenía claridad de este tema.

El 98.3\% (177 educadores de párvulos) declaró tener el cepillado de dientes incorporado en la rutina diaria. El 1.7\% que no realiza esta actividad (tres jardines), adujo no contar con baños adecuados para llevar a cabo esta actividad.

Del $98.3 \%$ que realiza cepillado, un $63.9 \%$ reportó realizar cepillado de dientes dos veces al día, un $18.9 \%$ lo realiza tres veces al día, y una vez al día el $16.6 \%$. Un $0.5 \%$ de la muestra no respondió.

Para evaluar si se utiliza cotidianamente dentífrico, se consultó si rutinariamente se solicita dentífrico a los apoderados, puesto que no es un insumo aportado por los establecimientos. Los resultados muestran que el $56.1 \%$ de los profesionales lo solicita, siendo esta solicitud igual para todos los niveles, no diferenciando por edad. Un $33.9 \%$ de los encuestados respondió que solicitaba pasta dental sólo desde el nivel medio mayor en adelante, es decir, desde los tres años. Un $7.2 \%$ no solicita pasta de dientes. Hubo un $2.8 \%$ que no respondió. Al profundizar en el tipo de 
pasta dental solicitada, el $86.7 \%$ declaró pedir pasta dental infantil a los padres y/o apoderados (consideradas pastas dentales infantiles aquellas con contenido de 400 a $500 \mathrm{ppm}$ de fluoruro). Un $5.6 \%$, solicita pasta dental de adultos. Un 3.3\% no responde. Un $4.4 \%$ responde en "otros".

Nivel de conocimientos. Cada dimensión de conocimientos medida fue ponderada diferencialmente, según aquellos conocimientos que se consideraron más pertinentes a la realidad de los educadores entrevistados (Tablas 2 y 3 ).

Los valores podían fluctuar entre 0 y 1 . El promedio general de conocimientos final fue de 0.69 , nivel bueno, para caracterizar al grupo de educadores investigado, con una desviación estándar de 0.12 y un rango de (0.26-0.92). El intervalo de confianza de $95 \%$ del nivel de conocimientos muestral fue de 0.67-0.70.

El propósito de establecer un nivel de conocimientos en prevención de caries y gingivitis en las parvularias y parvularios, fue instalar un estándar contra el cual contrastar, a futuro, los conocimientos adquiridos de modo de hacer comparables los resultados de próximas investigaciones.

Esto obligó a generar una escala para encasillar a los participantes de este estudio. Según lo estipulado con anterioridad, se presenta a continuación el resultado global obtenido con la muestra seleccionada.

Tabla 2. Nivel de conocimientos en prevención de caries y gingivitis de educadores de párvulos. Santiago, 2007.

\begin{tabular}{|l|l|c|c|l|}
\hline Nivel & Rangos & $\begin{array}{l}\text { Frecuencia } \\
\text { de resultados }\end{array}$ & Porcentaje & $\begin{array}{l}\text { Porcentaje } \\
\text { acumulado }\end{array}$ \\
\hline Insuficiente & $0-0.50$ & 13 & $7.2 \%$ & $7.2 \%$ \\
\hline Suficiente & $0.51-0.67$ & 60 & $33.3 \%$ & $40.5 \%$ \\
\hline Bueno & $0.68-0.84$ & 92 & $51.1 \%$ & $91.6 \%$ \\
\hline Muy bueno & $0.85-1.0$ & 15 & $8.3 \%$ & $100 \%$ \\
\hline
\end{tabular}

Al evaluar cada dimensión por separado y aplicarle la misma escala, se aprecia que la dimensión que obtuvo mejor evaluación fue gingi- vitis, y las que estuvieron más bajas fueron fluoruros e higiene bucal, lo que identifica áreas de trabajo para su refuerzo.

Tabla3. Nivel de conocimientos de educadores de párvulos, según dimensión evaluada.

\begin{tabular}{|l|l|l|l|}
\hline Dimensión & $\begin{array}{l}\text { Puntaje promedio } \\
\text { obtenido }\end{array}$ & $\begin{array}{l}\text { Intervalo de Confianza } \\
\mathbf{9 5 \%}\end{array}$ & Nivel \\
\hline Caries & 0.79 & $0.76-0.82$ & Bueno \\
\hline Dieta & 0.83 & $0.80-0.87$ & Bueno \\
\hline Fluoruros & 0.50 & $0.47-0.54$ & Suficiente \\
\hline Gingivitis & 0.90 & $0.86-0.93$ & Muy Bueno \\
\hline Higiene Bucal & 0.56 & $0.53-0.59$ & Suficiente \\
\hline
\end{tabular}

\section{DISCUSIÓN}

Los educadores de párvulos de los jardines infantiles JUNJI, se rigen por las normas espe- cíficas que dicta el Departamento Técnico de la institución, relativas a la educación y desarrollo de los párvulos, pudiendo representar un sesgo en relación a otros jardines infantiles. 
Los docentes que respondieron el instrumento de recolección de información (180), tienden a ser personas con una larga trayectoria profesional, de 20 o más años de ejercicio y con un promedio de edad de 45.8 años. Esto indica un conocimiento y manejo de los niños y niñas bajo su cuidado que ha podido ser aprendido y desarrollado, así como un conocimiento de la comunidad en la que está inserto el jardín infantil. Ambas situaciones pueden facilitar el trabajo promocional, ya que existe una conexión directa entre la comunidad, el establecimiento educacional y los padres, al menos en la educación escolar, en un intento por entregar educación en salud y promoción. Esto mismo se puede extrapolar a la educación pre básica, pues el objetivo es similar ${ }^{14}$, razón por la cual, es importante el grado de información, conocimientos y aplicación que ejercen los docentes en materias de salud.

No se obtuvo información que pudiera caracterizar a los 29 educadores de párvulos de la muestra que no respondieron el cuestionario, lo que es una de las limitaciones del estudio, ya que no se sabe cómo hubiera afectado el nivel de conocimientos.

En la evaluación, los docentes demostraron conocer conceptualmente qué se entiende por caries, y su etiopatogenia. En menor medida (53.3\%), reconocen el autocuidado como una forma de prevenir la enfermedad. Este dato es relevante, pues se sigue depositando en otra persona (profesional) la labor preventiva, aún cuando las medidas preventivas personales han demostrados ser eficientes ${ }^{15,16}$.

La mayoría de los docentes identifican a la consistencia, frecuencia de consumo y cantidad de azúcar refinada que contenga el alimento como importante en la producción de caries $(75.6 \%)$. Siendo estos elementos, factores modificables en cuanto a la conducta de selección de las personas, y por tanto intervenibles mediante estrategias promocionales y preventivas, resulta importante considerar un refuerzo en esta temática.

Existe conciencia mayoritaria de los beneficios del fluoruro en la prevención de la caries dental (97.2\%), posiblemente debido a campañas publicitarias constantes de dentífricos, sin embargo, existe poca claridad en relación a los medios, ya sea naturales o artificiales, por los que se puede obtener fluoruro(29.4\%), y, lo que para el propósito de un programa de prevención de caries es más relevante, la forma en que el fluoruro, por vía tópica, debe manejarse para obtener los máximos beneficios en los párvulos con el mínimo riesgo de fluorosis, a una edad en que el reflejo de deglución no se controla bien ${ }^{17-19}$. Se debe recalcar la edad de inicio del uso de dentífrico fluorurado y la cantidad a dosificar en la pasta, siendo relevante que la dispensación debiera ser hecha por un adulto para cautelar que no se use en exceso. Asimismo, la supervisión es necesaria para asegurarse que los menores escupan toda la espuma formada, de modo de evitar la deglución involuntaria de pasta dental, punto en el que sólo un $16.1 \%$ de los encuestados acertó. Un programa que involucre a educadores debe considerar la disponibilidad de personal docente y paradocente que pueda apoyar en la supervisión y control del cepillado con dentífrico fluorurado, de modo que todo el proceso se monitoree para asegurar un procedimiento protegido para los niños y niñas, lo que pudiera implicar involucrar a padres y/o apoderados como monitores de apoyo, previo una breve capacitación que explique razones del mismo y pasos a seguir.

Un elevado porcentaje de personas tiene incorporado el concepto de inflamación gingival $(92.2 \%)$ y sus causas $(88.3 \%)$, lo que favorece el realizar actividades de prevención en esta materia, pues la información ya existe.

La edad de inicio de la higiene bucal fue respondida adecuadamente por el $74.4 \%$ de las personas encuestadas, un $14.9 \%$ mencionó como tal el año de vida, mientras que un 6\% la ubicó a los dos años. Al año de vida ya están expuestos al medio bucal los incisivos superiores e inferiores temporales (ocho piezas dentarias en promedio), mientras que a los dos años, existen, en promedio, 14 piezas dentarias expuestas al medio, y el niño o niña, ya se encuentra en el período defini- 
do como ventana de la infectividad ${ }^{20}$, por lo que aclarar este punto a las educadoras y educadores para que difundan esta información a los padres y apoderados, se considera importante.

Se aprecia una oportunidad en el hecho de que en estos jardines está incorporada la rutina de cepillado. Desde la visión cuantitativa y de procesos, es necesario avanzar hacia la cualitativa para evaluar cómo se está realizando esta práctica y mejorarla si fuere necesario, puesto que ya existe el hábito de efectuar la frecuencia mínima adecuada de cepillado en el jardín, en casi $2 / 3$ de los jardines estudiados.

Según la escala elaborada para medir el nivel de conocimientos, el promedio obtenido para el grupo fue de 0.69 , catalogado como bueno en este estudio. Estos resultados permiten augurar un buen trabajo intersectorial, si se aprovecha la instancia de los establecimientos promotores de salud ${ }^{21}$ para instalar estrategias de promoción y prevención en salud bucal de párvulos, pues la cantidad de información que sería necesario instalar se focaliza en ciertos ítems, los que sería deseable reforzar, para nivelar los conocimientos actuales. Las dimensiones que requieren refuerzo corresponden al uso racional de fluoruros, e higiene bucal.

En cuanto a las prácticas habituales de prevención, se obtuvo que, en relación al control de la dieta, por normativa institucional, no se permite a los párvulos traer golosinas ni colaciones desde sus hogares, limitándose a las comidas regulares que se ofrecen en el establecimiento, lo que favorece el control de la frecuencia de ingesta de alimentos cariogénicos, siendo una práctica consistente con las recomendaciones internacionales de prevención de caries.

Considerando como limitación que la información recabada se obtuvo mediante un cuestionario autoaplicado, de tipo voluntario y anónimo, en el que no hubo posibilidad de explicar dudas a los profesionales, y sin la certeza de la individualidad de las respuestas, resulta pertinente sugerir la estandarización de los conocimientos de prevención de caries y gingivitis en el preescolar, durante el período de reclutamiento e inducción del personal docente en la Junta Nacional de Jardines Infantiles. Esto es mediante examen del nivel de conocimientos al ingresar, entrega de documentos educativos e, idealmente, refuerzo realizado en capacitación anual por odontólogo en caso de determinarse como necesario si el nivel detectado fuese insuficiente (mediante apoyo de profesionales de universidades, organismos públicos, establecimientos de atención primaria), examen posterior a la capacitación para medir el cambio producido.

Recibido: 30 julio 2008 Aprobado: 26 agosto 2008

\section{REFERENCIAS}

1. MINISTERIO DE SALUD. Diagnóstico de Situación de Salud Bucal. Departamento de Salud Bucal; 2003.

2. MINISTERIO DE SALUD. Diagnóstico Nacional de Salud Bucal del Niño de 6 años, Chile. Subsecretaría de Salud Pública. Santiago: MINSAL; 2007.

3. SCOTTISH INTERCOLLEGIATE GUIDELINES NETWORK. Prevention and management of dental decay in the pre-school child.A national clinical guideline. November 2005 [citado 29 ago 2008]. Disponible en URL: http:// www.sign.ac.uk

4. BRAVO M, LL J, CORTÉS F, CASALS E. Encuesta de Salud Oral en preescolares en España 2007. RCOE, 2007, Vol. 12, No3, 143-168.

5. JUNTA NACIONAL DE JARDINES INFANTILES. BALANCE DE GESTIÓN INTEGRAL AÑO 2007

6. MINISTERIO DE PLANIFICACIÓN Y COOPERACIÓN. PNUD (Chile).Desarrollo Humano en las Comunas de Chile. Temas de Desarrollo Sustentable. Santiago; 2000.

7. FEATHERSTONE JDB. Caries prevention and reversal based on the caries balance. Pediatr Dent 2006; 28: 128-132. 
8. TOUGER-DECKER R, MOBLEY CC. American Dietetic Association Position of the American Dietetic Association: oral health and nutrition. J Am Diet Assoc. 2007 Aug;107(8):141828.

9. MENTES A, ATUKEREN J.A study of manual toothbrushing skills in children aged 3 to 11 years. J Clin Pediatr Dent. 2002 Fall; 27(1): 91-4.

10. BOROTO O, MORA C, ARAÚJO M, LÓPEZ R. Nivel de conocimiento de Educación para la Salud en Ortodoncia de trabajadoras de círculos infantiles. Rev Cubana Ortod 2001:16(2): 83-9.

11. YAHYA B N, SOLMAZ S. The knowledge, approach and function of pediatricians in prevention of caries in Teheran. J Indian Soc Ped Prev Dent September 2004; 22 (3): 148-153.

12. DUNNING B, CAHALAN D. By-Mail vs. Field Self-Administered Questionnaires: An Armed Forces Survey . The Public Opinion Quarterly, Vol. 37, No. 4 (Winter, 1973-1974), p. 618-624.

13. WILKINS III JR, WUESTON WD, MAC CRAWFORD J, STEELE LL, GERKEN DF. Mixed mode survey of female veterinarians yields high response rate. Occup Med. Vol $47 \mathrm{~N}^{\circ}$ 81997, 458-462.

14. SMITH BJ, POTTS-DATEMA W, NOLTE AE. Challenges in teacher preparation for school health education and promotion. Promot Educ. 2005;12(3-4):162-4.
15. BROADBENT JM, THOMSOM WM, POULTON R. Oral health beliefs in adolescence and oral health in young adulthood. J Dent Res 2006 Apr;85(4):339-43.

16. RONG WS, BIAN JY, WANG WJ, WANG JD. Effectiveness of an oral health education and caries prevention program in kindergartens in China. Community Dent Oral Epidemiol. 2003 Dec;31(6):412-6.

17. WARREN J, LEVY SM. A review of fluoride dentifrice to dental fluorosis. Pediatr Dent 1999; 21:265-271.

18. PENDRYS DG. Risk of enamel fluorosis in nonfluoridated and optimally fluoridated populations: considerations for the dental professional. J Am Dent Assoc 2000; 131 (6):746-755.

19. MARINHO VC, HIGGINS JP, SHEIHAM A, LOGAN S. Fluoride toothpastes for preventing dental caries in children and adolescents. Cochrane Databases Syst Rev 2003; 1: CD002278.

20. CHAN KM, KING N M, KILPATRICK NM.Can infants catch caries? A review of the current evidence on the infectious nature of dental caries in infants. New Zealand Dental Journal March 2005; 101 (1): 4-11.

21. WORLD HEALTH ORGANIZATION. School and youth health. (citado 29 jul 2008). Disponible en URL: http://www.who.int/school_ youth_health/en/

Usted puede comentar éste y otros artículos publicados en la Revista Chilena de Salud Pública, enviando un correo electrónico a revistasp@med.uchile.cl 